\begin{tabular}{ll} 
Soft and sticky & 112 \\
\hline Working the mechanics & 112 \\
\hline An array of possibilities & 113 \\
\hline Box 1: A niche market & 113 \\
\hline Heart of the matter & 114 \\
\hline
\end{tabular}

\title{
Where stem cells call home
}

\author{
Vivien Marx
}

By building engineered microenvironments, scientists probe the many talents of stem cells.

Stem cells seem to have superhero powers that allow them to continuously self-renew and grow and to differentiate into a variety of cell types. Using tuned materials, microfluidics and three-dimensional cell culture, researchers seek to mimic the microenvironment around stem cells-the nichethat shapes stem cell behavior in ways that have not yet been completely deciphered.

Engineered stem cell niches are controlled environments created to replicate the in vivo niche more closely than traditional cell culture can. Scientists can study cell proliferation, self-renewal, differentiation or quiescence and can listen in on the intense communication between stem cells and their surroundings. Ultimately, these efforts could reveal better ways to culture stem cells and guide the scaling up of stem cell production.

In this field, interdisciplinary teams of stem cell biologists and engineers self-

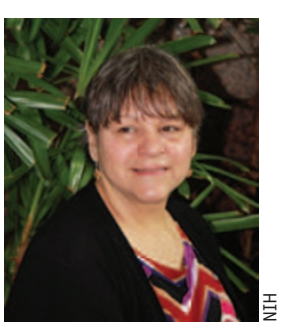

Figuring out the needs of stem cells is "a very complex dance," says Rosemarie Hunziker. as emble, not unlike the building blocks of a new biomaterial, says Rosemarie Hunziker, program director at the National Institute of Biomedical I m a ging and Bioengineering. Ideally, researchers would like to recreate all the details of the in vivo stem cell niche. Although that level of detail is hard to achieve for any lab, collaborations help them identify the necessary and sufficient factors to achieve their ends.

As an example, making red blood cells from stem cells in vitro requires different environmental cues than making white blood cells, so scientists must mimic and control the relevant factors. "It's a very complex dance you have to execute here, to figure out what cell type you want, and what's important for that," she says.

One basic hurdle is that the many subdisciplines of stem cell biology differ dramatically in their definition of the niche, says Arthur Lander from the University of California at Irvine ${ }^{1}$. What also intrigues scientists is that stem cells are often, but not always, able to differentiate into many kinds of cells. And there is even diversity among stem cells in a niche. Yet he believes that common themes can unify the field. One such theme is achieving control over stem cell behavior.

Some control of stem cell behavior may be hardwired, but much of it is likely mediated by feedback from the stem cell's environment. "The challenges are really to find out what is being controlled, how it is being controlled and for what purpose is it being controlled," he says.

\section{Not a doghouse}

To architects, niches are nooks in buildings, points out Harvard University stem cell biologist David Scadden ${ }^{2}$. Field biologists use the term to describe where an organism resides and reproduces. In French, 'niche' means 'doghouse'. "So, the grand position of the stem cell in popular concepts of science is appropriately humbled by the cells dwelling in a place where they might awaken with fleas," Scadden notes.

But, he says, the stem cell niche, first described over three decades ago by Ray Schofield as the "cellular environment which retains the stem cell" 3 , is anything but a modest abode. Rather, it is a dynamic environment with signaling cues that let stem cells persist, grow, change their

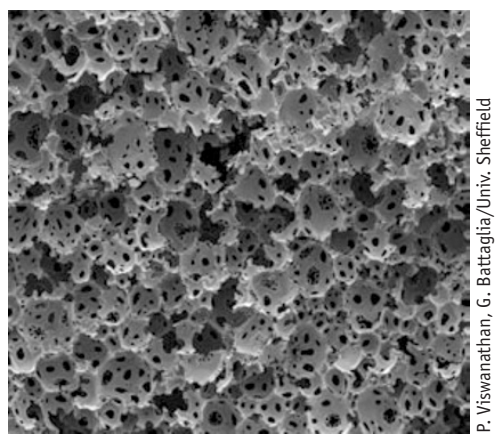

An engineered porous foam mimics the body's sticky extracellular matrix. This scanning electron micrograph reveals the pockets where human stem cells adhere when the foam has the preferred degree of stickiness.

fate and react to the physiological needs of the organism.

In a cross-disciplinary project, Scadden, along with Harvard engineering colleagues David Mooney, David Weitz and Robert Westervelt, is building the hematopoietic stem cell niche. The goal is a threedimensional model of the stem cell niche in the bone marrow, complete with environmental cues. This system will shed light on blood stem cells and blood cell development and pathologies, all of which are hard to study in vivo. Down the road, tissue engineers will be able to use these models for biomedical applications.

Researchers know plenty about components and signals in the blood stem cell niche 4 . "We now are trying to engineer a system where we can present these things in a very controllable manner," says Mooney. The project draws on cell biology, materials engineering and microfluidics. Mammalian cells, except for circulating blood cells, are surrounded by scaffolds made of the extracellular matrix (ECM), which impart much chemical information to stem cells, he says. "The 


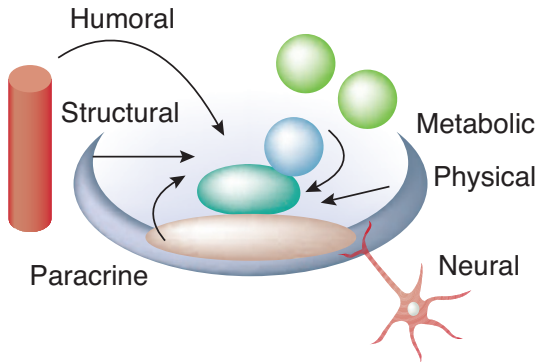

The stem cell niche interacts mechanically and chemically with stem cells. Reprinted from ref. 2 .

idea here is that we will use the microfluidics to traffic cells and to coat cells with materials."

Several research groups are testing different chemistries to approximate the ECM in vitro. As Mooney explains, the ECM was once considered an inert support structure, but research has revealed it to be a signaling hub, with a critical role in the niche for developing and maturing stem cells $s^{5}$. "If they can bind to the material, they can in many ways read the mechanical properties, and that dictates a lot of their decision making," he says.

\section{Soft and sticky}

Hydrogels are a class of materials with high water content, similar to the ECM. The

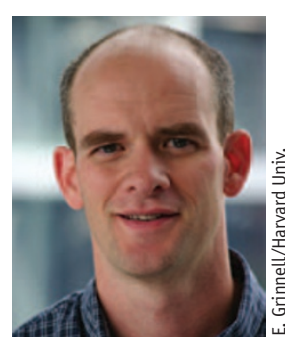
stickiness of these polymers has to be chemically tuned. "We want the cells to stick only in the manner that we specifically build into the material," Mooney says. To allow manipulation

"The idea here is that we will use the microfluidics to traffic cells and to coat cells with materials," says David Mooney. of individual stem cells, he works with polymers based on the natural polysaccharide alginate.

When the scientists want to test a specific hydrogel, they use microfluidics: in the microfluidic device, alginate, water, calcium chloride, oil and a surfactant flow in through separate channels. The calcium cross-links the alginate such that it forms a polymeric network, and the other ingredients help steer the reaction in which stem cells are coated with a thin layer of the synthetic ECM. "You take cells and put them through a microfluidic system in a manner that you get individual cells surrounded by precursors to the gel," says Mooney. Each stem cell is engulfed in its own individual droplet. Next, the droplet is exposed to conditions under which the gel solidifies around the cell.

Every step involves plenty of engineering challenges, including the need for different gelation methods and to direct flow streams, says Weitz. When the device is in operation, "we use multiple streams to control where the reaction takes place to ensure that the nozzle does not become clogged."

The cells' coating has to be compatible with the microfluidics and designed according to biological findings from Scadden's lab and others. The team combines the components in the coating in different ways as they build and test the niche. They want to determine, says Mooney, what to "build in at a minimum to maintain the stem-ness of the hematopoietic stem cell.” Each task along the way takes collaboration. "To be successful in this type of space, you have to be very, very interactive," he says.

\section{Working the mechanics}

Other engineering efforts to build in vitro niches focus on different ECM traits. Bioengineer Adam Engler at the University of California at San Diego (UCSD) is developing what he calls "smart materials" for a synthetic ECM. In 2009, he received a US National Institutes of Health (NIH) New Innovator award, which is intended for ideas that, in the NIH's words, "have the potential for unusually high impact."

Whereas most hydrogels are more or less static, in vivo the niche's ECM stiffens over time, which is a shift that an engineered matrix must mirror, Engler says. He is developing a hybrid hydrogel that changes in this way. As an engineer, he concedes that "this is difficult chemistry to accomplish," but the biologist in him says that research findings call for this ECM chemistry. The niche is more than just the cell types and growth factors present.

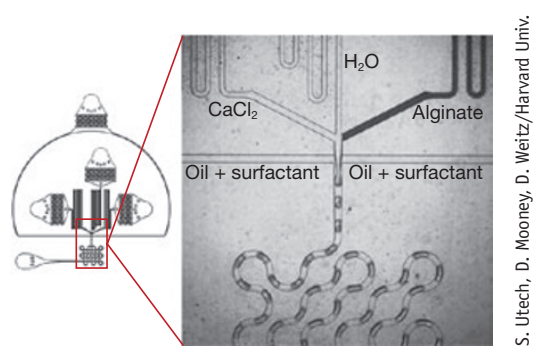

To create an in vitro stem cell niche, scientists have built microfluidic devices with flow streams to mix components and then to envelope stem cells in a tailored polymer. 


\section{BOX 1 A NICHE MARKET}

If artificial stem cell niches eventually become a commercial platform to control stem cell differentiation, they must offer fine levels of control and "be easy to use," says Nick Asbrock, product manager for stem cell and cell biology at EMD Millipore, which sells stem cells, growth and differentiation media, and antibodies for cell characterization. A new offering is a microfluidic perfusion system developed by a University of California, Berkeley spin-out, CellASIC, which Millipore acquired. "You can program the computer to tell it exactly what to add to the cells in the dish," he says. Instead of surrounding the cells with stagnant medium, the system allows controlled and timed release of growth medium.

Companies use different ways to drive stem cells to a specific fate, says Marcus Yeo, CEO of Definigen, a University of Cambridge spin-out. Engineered niches could become an asset, but they must mimic the in vivo signaling environment adequately. "It's how specific you can be in terms of adding media factors, growth factors that can really duplicate the key stages of mammalian development," he says. "The better you become at that, the more effective the cells will be."

In Definigen's labs, scientists take adult fibroblast cells, reprogram them into stem cells and then differentiate them into liver cells or pancreatic cells for use in academic and drug discovery research. Yeo believes his company has reproducible approaches to coax out stem cell-niche interaction and standardized stem cell production. "We don't want one batch to be good and one batch to be appalling," he says.

Researchers who are engineering stem cell niches need well-characterized media, says Erik Hadley, senior scientist in research and development at Stemcell Technologies, which is a British Columbia Cancer Agency spin-out. His company supplies reagents for scientists building niches, including a maintenance medium that lets them focus on stem cell-niche interaction. Licensed from the University of Wisconsin, the medium has been formulated with only a minimal set of components.

To accompany this offering, the company is starting to distribute a surface coating from Primorigen Biosciences. His company has tuned the simplified medium to work in combination with this coating. "It's basically a simple surface, a simple mimic of the niche, and will be offered in conjunction with the simplified media," Hadley says. The combined reagents can, for example, be used by teams testing niches and different spatial orientations, such as in threedimensional experiments.

As engineered systems mature and can be scaled up, costs rise too. Resodyn Corporation, a company that develops coating and mixing systems, aims to make large-scale stem cell culture more economical. "The growth factor molecules cost too much, and you have to use too much of them," says Resodyn chief bioengineer Todd McAdams. McAdams is studding polymer beads with select growth factors to offer them to stem cells as bound molecules. The stem cells cannot internalize these growth factors, which allows their reuse. "It helps stimulate the stem cell in the way that you want to," he says. Although the medium still needs soluble factors, he hopes these bead-bound growth factors could deliver cost savings for stem cell culture.

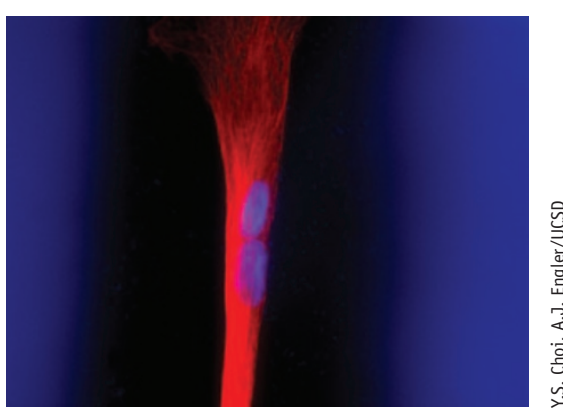

Stem cells (red) derived from human fat grow on the stiffer regions of a mechanically patterned hydrogel. As they grow, the stem cells fuse and form cells with multiple nuclei (blue ovals) as seen in typical muscle cells.

Researchers also need to mind the chemical and mechanical qualities of the stem cell niche, he says. "As a matrix biologist, I believe that there is a tremendous amount of signaling that occurs solely from the matrix itself, which can direct stem cell fate." Cells actively sense the matrix around them and its properties, all of which factor into the stem cell's behavior.

Along with colleagues at the University of Sheffield and the Sanford Consortium for Regenerative Medicine, Engler and his team recently developed a porous synthetic $\mathrm{ECM}^{6}$. The scaffold has tunable physical and chemical properties to support stem cell growth and development. The ECM is prepared through a process called high internal phase emulsion templating, which creates a foamed surface that has pockets of varying stickiness. The team studied where proteins and stem cells are more likely to adhere in this ECM. The scientists believe its heterogeneous surface chemistry better reflects adhesion in the natural ECM over some other synthetic ECMs.

\section{An array of possibilities}

Engler follows other approaches to create arrays of potential niches made with synthetic ECMs. His colleagues at UCSD adapted conventional printing technology to dot a substrate with defined compositions of matrix proteins. Stem cells can be bound to each dot, allowing scientists to study their properties in many different matrix-based niches. This technology was commercialized by a company called MicroStem. Stem cell niche engineering has led to a number of commercial ventures (Box 1).

Bioengineer Matthias Lutolf at the École Polytechnique Fédérale de Lausanne (EPFL) also applies printing techniques, meshing hydrogel engineering with robotics to generate several hundred up to

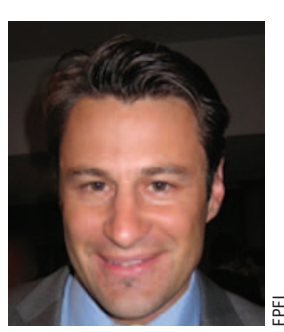

High-throughput artificial niche screening systems require investment in instrumentation, says Matthias Lutolf. 1,000 printed artificial niche candidates in a single experiment. This method can help identify suitable microenvironments to maintain adult stem cells in their multipotent state, he says. "In this context, we are mainly interested in identifying artificial niches for hematopoietic stem cells, with the goal of expanding these cells for transplantation."

The robot is a standard DNA spotter or automated pipettor that precisely 
mixes chosen soluble stem cell niche components, such as growth factors or ECM proteins, in nanoliter volumes. The mixtures are then dispensed in high throughput onto a substrate where they generate spots with a wide range of defined properties. These spots are niches that can then be tested for their effects on stem cell function. Lutolf has developed a two-dimensional format and is currently experimenting with a three-dimensional one as well ${ }^{7}$.

"Of course, these high-throughput artificial niche screening systems are somehow a brute-force approach that is not so trivial to implement, and it also requires some investment in instrumentation," he says. For example, cell imaging with timelapse microscopy before and after culture requires powerful automated imaging stations.

High-throughput stem cell niche engineering helps experimenters mainly because niches are complex microenvironments with possibly hundreds of biochemical and biophysical cues acting in concert, perhaps synergistically, to control stem cell fate, he says. "Often, these signals have not even been discovered, so we don't know what to engineer."

Lutolf and colleagues are also combining hydrogel engineering with microfluidics. Similarly to the Harvard team and others, he believes controlled delivery of signals to the niche allows scientists to modulate the properties of an artificial stem cell niche during an experiment. A modulated niche better reflects the in vivo situation because, for example, in an organism the number of stem cells and niches changes.
Researchers have also noted gradients across a stem cell niche, with stem cells exposed to different signals in different locations in the niche, which shapes the way the cells divide, he says. "Having the possibility to engineer such spatial complexity in culture is very powerful," he says. Microfluidics gives scientists ways to manipulate single stem cells to better understand behavior across a diverse population of stem cells.

At the University of Michigan, bioengineer Shuichi Takayama and his team are taking niche printing underwater as they micropattern cells and niches under completely aqueous conditions. Two cell culture media containing different polymers are used in the patterning. "Because the process is completely underwater, the cells are happy," Takayama says. He is also experimenting with microchannel-based systems as well as three-dimensional hanging-droplet cell culture plates.

\section{Heart of the matter}

Disappointment with clinical trials in cellular therapy has focused attention on the stem cell niche and niche engineering. Even after many years of clinical trials, cardiac cell therapy has not yielded positive results. In vitro and animal studies seemed to indicate that injected stem cells and various added factors could induce cells to differentiate into heart muscle cells to replace those damaged in a heart attack, says Jay Schneider from the University of Texas Southwestern Medical Center. Ten years on, it has become clear that the trials "put the clinical cart before the scientific horse," which has reinforced research into a possible stem cell niche in the heart.

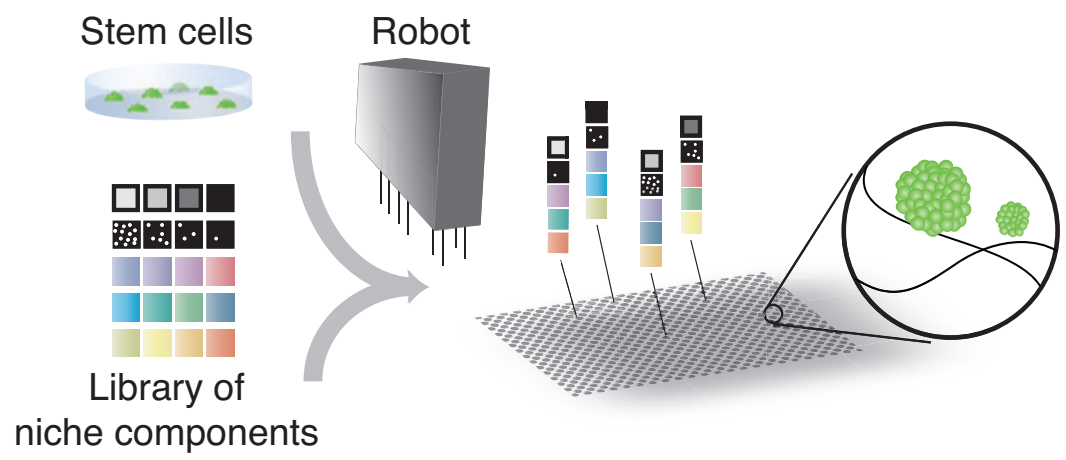

Hydrogel engineering combined with robotics can generate several hundreds to a thousand printed artificial niche candidates in one experiment. 

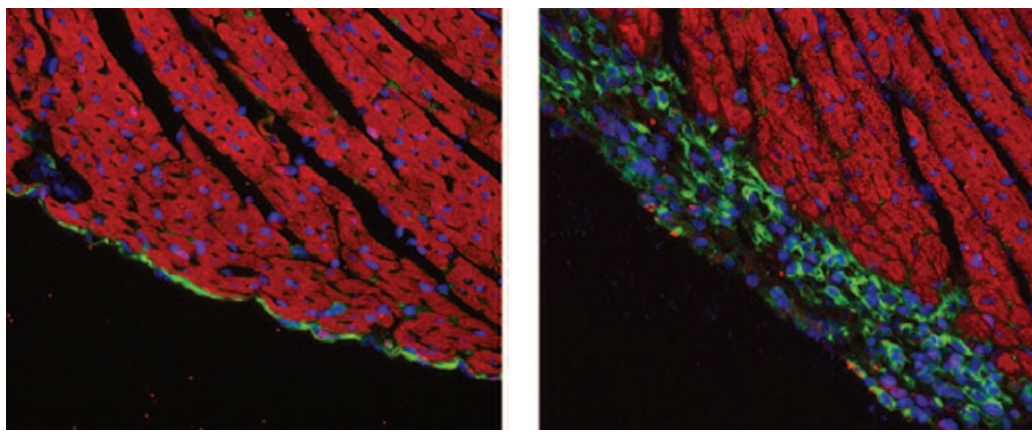

The adult heart may have a stem cell niche in the layer around the heart (left; green). In reaction to injury, the cell layer around the heart thickens (right).

"What the heart really needs is a hippocampus," he says, in which neurons are born from neural stem cells along with sprouting microcapillaries. Although the adult heart lacks a true stem cell niche, it appears to have a vascular niche.

"You need to generate blood vessels and muscle cells coordinately, to develop at the same time, so that every new muscle cell that you make has a bunch of capillaries around it supplying nutrients and oxygen and removing waste products." Upon injury, the one-cell-thin layer of cells around the heart thickens and scar tissue is formed. The microenvironment of this layer, the epicardium, appears to have stem cell niche attributes. If researchers could turn back the clock in this region to induce heart cell development rather than scar-tissue formation, "that would be key," he says. But that approach remains a "huge engineering problem."
Along with Scadden, Schneider heads a working group on microenvironmental control of progenitors in the National Heart, Lung, and Blood Institute's Progenitor Cell Biology Consortium. For now, given the heart's complexity, Schneider believes that successful niche engineering is more likely to first succeed in the hematopoietic stem cell niche.

1. Lander, A.D. et al. BMC Biol. 10, 19 (2012).

2. Scadden, D.T. Nature 441, 1075-1079 (2006).

3. Schofield, R. Blood Cells 4, 7-25 (1978).

4. Lo Celso, C. \& Scadden, D.T. J. Cell Sci. 124, 3529-3535 (2011)

5. Hynes, R.0. Science 326, 1216-1219 (2009).

6. Viswanathan, P. et al. J. Am. Chem. Soc. 134, 20103-20109 (2012).

7. Gobaa, S. et al. Nat. Methods 9, 949-955 (2011).

Vivien Marx is technology editor for Nature and Nature Methods.

(v.marx@us.nature.com). 